\title{
BMJ
}

\section{Helicobacter pylori test and treat versus proton pump inhibitor in initial management of dyspepsia in primary care: multicentre randomised controlled trial (MRC-CUBE trial)}

\author{
Brendan C Delaney, professor of primary care, ${ }^{1}$ Michelle Qume, research fellow, ${ }^{1}$ Paul Moayyedi, professor \\ of gastroenterology, ${ }^{2}$ Richard F A Logan, professor of epidemiology, ${ }^{3}$ Alexander C Ford, lecturer, ${ }^{4}$ \\ Cathy Elliott, research associate, ${ }^{3}$ Cliodna McNulty, consultant microbiologist, ${ }^{5}$ Sue Wilson, professor of \\ epidemiology, ${ }^{1}$ F D Richard Hobbs, professor of primary care and general practice
}

${ }^{1}$ Primary Care Clinical Sciences, University of Birmingham, Birmingham B15 2T

${ }^{2}$ McMaster University, Hamilton, ON, Canada

${ }^{3}$ Division of Epidemiology and Public Health, University of Nottingham, Nottingham

${ }^{4}$ Centre for Gastrointestinal Disease, Leeds General Infirmary, Leeds

${ }^{5}$ Health Protection Agency Primary Care Unit, Gloucester

Correspondence to: B Delaney b.c.delaney@bham.ac.uk

doi:10.1136/bmj.39479.640486.AE

\section{ABSTRACT}

Objective To determine the cost effectiveness of Helicobacter pylori "test and treat" compared with empirical acid suppression in the initial management of patients with dyspepsia in primary care.

Design Randomised controlled trial.

Setting 80 general practices in the United Kingdom. Participants 699 patients aged 18-65 who presented to their general practitioner with epigastric pain, heartburn, or both without "alarm symptoms" for malignancy.

Intervention $\mathrm{H}$ pylori ${ }^{13} \mathrm{C}$ urea breath test plus one week of eradication treatment if positive or proton pump inhibitor alone; subsequent management at general practitioner's discretion.

Main outcome measures Cost effectiveness in cost per quality adjusted life year (QALY) (EQ-5D) and effect on dyspeptic symptoms at one year measured with short form Leeds dyspepsia questionnaire.

Results 343 patients were randomised to testing for $H$ pylori, and 100 were positive. The successful eradication rate was $78 \%$. 356 patients received proton pump inhibitor for 28 days. At 12 months no significant differences existed between the two groups in QALYs, costs, or dyspeptic symptoms. Minor reductions in costly resource use over the year in the test and treat group "paid back" the initial cost of the intervention.

Conclusions Test and treat and acid suppression are equally cost effective in the initial management of dyspepsia. Empirical acid suppression is an appropriate initial strategy. As costs are similar overall, general practitioners should discuss with patients at which point to consider $H$ pylori testing.

Trial registration Current Controlled Trials ISRCTN 7644265 .

\section{INTRODUCTION}

Dyspepsia is a common problem ${ }^{12}$; in the United Kingdom, 3\% of the population take long term prescribed drugs for dyspepsia at an annual cost of $£ 500$ million ( $€ 663 \mathrm{~m} ; \$ 983 \mathrm{~m}$ ), and 450000 patients have upper gastrointestinal endoscopy each year. ${ }^{3}$ The cost effectiveness of strategies for managing dyspepsia have been studied in several randomised controlled trials and summarised in a Cochrane review, ${ }^{4}$ now widely translated into UK, European, and US guidelines. ${ }^{5-7}$ Endoscopy is not as cost effective as either empirical acid suppression or testing for and treating Helicobacter pylori infection ("test and treat"). ${ }^{8}$ An economic model has suggested that test and treat is cost effective, with an incremental cost effectiveness ratio of $£ 63$ per month free of symptoms over five years, compared with intermittent proton pump inhibitor. ${ }^{9}$ However, whether test and treat is an appropriate first line strategy has remained less clear. The 2004 guideline from the National Institute for Clinical Excellence (NICE) on the management of dyspepsia in adults recommended that either test and treat or proton pump inhibitor was appropriate initial management for dyspepsia in primary care, on the grounds of a lack of evidence as to their relative cost effectiveness. ${ }^{10}$ NICE also proposed that the initial management of patients with predominant epigastric pain and predominant heartburn should be the same, on the grounds that symptom patterns do not reliably predict underlying pathology.

Test and treat can be compared with empirical prescribing, with randomisation at the level of the strategy, following both $H$ pylori positive and negative patients in a pragmatic trial, or after $H$ pylori testing, randomising only $H$ pylori positive patients and comparing them with placebo. Two trials in which patients were randomised after testing to either eradication of $H$ pylori or treatment with a proton pump inhibitor and placebo antibiotics have been completed. The Cadet-Hp study, set in primary care in Canada, showed significantly fewer recurrent dyspeptic symptoms, on the basis of "absence of symptoms"$72 \%$ of patients on eradication treatment had dyspepsia at one year compared with $85 \%$ of those on proton pump inhibitor alone. ${ }^{11}$ Test and treat also saved money compared with proton pump inhibitor and placebo antibiotics, with an incremental net monetary 
benefit of \$C45 (£23) (90\% confidence interval -10 to 100). ${ }^{12}$ An additional unpublished UK study has also shown a similar benefit in favour of test and treat compared with placebo. ${ }^{13}$ However, as both of these studies involved randomisation after testing, they are not generalisable to the decision to test, as the behaviour of patients may change with the knowledge of a positive or negative test. One small study of 219 patients in Italy randomised at the level of the strategy. ${ }^{14}$ It suggested that test and treat was more effective than empirical acid suppression as an initial management strategy. However, the study lacked an economic analysis, took place in the gastroenterology clinic setting, and had an unusually high $H$ pylori infection rate $(61 \%)$; the principal outcome was symptomatic relapse, at which point patients automatically had endoscopy.

A further problem has been the shifting role of heartburn in the definition of functional dyspepsia. In 1999 the definition of functional dyspepsia was revised by the Rome II international working party, ${ }^{15}$ to exclude patients with "predominant heartburn" (it was revised again in 2006: Rome $\mathrm{III}^{16}$ ). Definitions of uninvestigated dyspepsia, based on the definition of functional dyspepsia and using symptom patterns, had been shown to be poorly predictive of particular organic disease.$^{17}$ In particular, patients with heartburn in primary care are just as likely to have a peptic ulcer as oesophagitis, owing to the poor negative predictive value of heartburn for peptic ulcer disease in uninvestigated patients and justifying the approach taken by NICE. ${ }^{18}$ A pragmatic trial was therefore needed to determine whether the effect of $H$ pylori eradication treatment is diminished in patients with predominant heartburn and whether these patients should be excluded at this early stage in management.

The primary aim of the MRC-CUBE (carbon-13 urea breath test and eradication) study was to

\section{Exclusion criteria}

- Knowledge of previous test for Helicobacter pylori

- Dysphagia

- Weight loss

- Haematemesis or melaena

- First degree relative with gastric cancer

- Age 55-65 with continuous as opposed to episodic epigastric pain or a total history of any dyspeptic symptoms of less than one year at presentation (in accordance with the current NHS two week suspected cancer referral guidelines)

- Knowledge of endoscopically proved peptic ulcer disease or severe oesophagitis (who should all receive either eradication treatment (ulcer) or proton pump inhibitor (oesophagitis))

- Pregnant women

- Patients who were unable to give informed consent

- Patients who were taking regular non-steroidal anti-inflammatory drugs (who might have ulcers induced by these drugs) or had started aspirin $75-150 \mathrm{mg}$ in the previous three months

- Patients allergic to study drugs

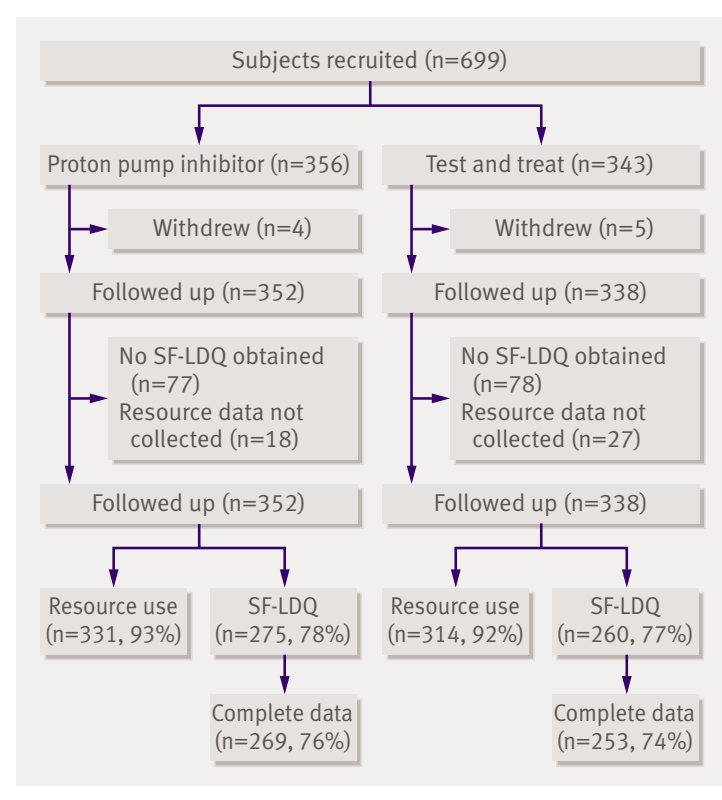

Fig 1| Flow of participants through study

determine the cost effectiveness of an H pylori test and treat strategy compared with empirical acid suppression for dyspepsia in primary care. The secondary aim was to determine the effect on dyspeptic symptoms in subgroups of patients with predominant heartburn and predominant epigastric pain.

\section{METHODS}

\section{Participants}

This was a multicentre, primary care based, randomised controlled trial with randomisation at the level of the individual patient. We recruited participants from 80 practices in England between January 2003 and January 2005. We initially recruited practices through the Midlands Research Practices Consortium, by writing to all 500 member practices, and from practices in Leeds and Nottingham that had previously participated in research with us. We supported practices with an initial training meeting, a training DVD, streaming video from the website, regular calls and visits from research staff, feedback on practice recruitment performance on a monthly basis, and a newsletter. In addition, in practices that used the EMIS electronic patient record, we configured the "pop-up" reminder system to flag patients who fitted the study criteria.

Eligible patients were those aged 18-65 years who consulted their general practitioner with dyspepsia. We defined dyspepsia broadly, according to the Rome I criteria, ${ }^{15}$ as a symptom complex consisting of one or more recurrent symptoms of pain centred in the upper abdomen, heartburn, acid regurgitation, nausea, or fullness and early satiety, of more than four weeks' duration. The box shows the exclusion criteria. General practitioners identified suitable patients during routine consultations. After giving consent, participants were randomised by use of a centralised, secure web based clinical trial management system 
(MidReC-en) to conceal allocation and verify compliance with the study procedures. Real time, online randomisation was computer generated, stratified by practice, and blocked by permuted blocks of size two, four, and six. After verification of the baseline data collection and consent, the system issued a unique study identification number and the treatment allocation. Online prompting for the breath test and study procedures was also provided during the data collection. We ran a telephone back-up system from the trial office. Participants already taking a proton pump inhibitor were eligible if they could be switched to antacid alone for two weeks.

\section{Interventions}

Patients randomised to test and treat had a ${ }^{13} \mathrm{C}$ urea breath test for $\mathrm{H}$ pylori, with sampling by the practice nurses and analysis of triplicate samples by a central reference laboratory (Institute of Human Nutrition, Southampton General Hospital). We used orange juice before the test to delay gastric emptying, and samples were taken 20 minutes after the ingestion of urea. We used an accepted delta value of $3.5 \%$ as the cut-off point to define positivity, with a performance of more than $99 \%$ sensitivity and specificity. All practice nurses had received training in the standard test protocol. Patients who tested positive were offered $H$ pylori eradication with one week of omeprazole $20 \mathrm{mg}$ once daily, clarithromycin $250 \mathrm{mg}$ twice daily, and metronidazole $400 \mathrm{mg}$ twice daily, followed by three weeks of omeprazole $20 \mathrm{mg}$ once daily. Patients who tested negative received omeprazole $20 \mathrm{mg}$ once daily for four weeks. Patients randomised to empirical acid suppression received omeprazole $20 \mathrm{mg}$ once daily for four weeks. Patients given eradication treatment were asked to attend for a follow-up breath test at 12 weeks, the result of which remained blinded until the end of the study.

After the initial intervention, general practitioners were free to manage patients with recurrent symptoms in both groups as they wished, with the caveat that $H$ pylori eradication treatment was specifically excluded for the 12 months of follow-up, unless the patient had endoscopically proved peptic ulcer disease.

\section{Outcome measures}

The primary outcome was cost effectiveness, determined as the incremental cost effectiveness ratio (difference in costs divided by difference in effect). We determined the difference in health services dyspepsia related costs by application of national reference costs to individual units of resource consumption (prescribing, consultations, interventions, and investigations). We determined the difference in effect as the difference in the absolute number of patients with no dyspeptic symptoms measured by the short form Leeds dyspepsia questionnaire, a validated community based measure that also includes a question on "predominant symptom." ${ }^{19}$ We also calculated an additional measure of effect-quality of life as measured with the EuroQol EQ-5D. ${ }^{20}$ This was to enable cost effectiveness to be expressed as cost per quality adjusted life year (QALY).

Secondary outcome measures were change in the score on the short form Leeds dyspepsia questionnaire, resource use, and patient satisfaction assessed with the consultation satisfaction score (subscales 1 and 2, general and professional care). ${ }^{21} \mathrm{We}$ calculated costs of managing dyspepsia by applying 2005 national reference costs to the units of resources used (tables 1 and 2). ${ }^{22-25}$ The practice nurses interviewing the patients completed the baseline EQ-5D and symptom scores. Participants saw the practice nurse at one year, for completion of the final outcome EQ-5D, symptom score, satisfaction score, and dyspepsia related resource use. Research staff used a 10\% independent review to validate the accuracy of the resource use data collected by the nurses and entered online. Participants who did not attend were initially sent a postal questionnaire and subsequently contacted by telephone. We did not collect data on indirect costs or use of over the counter drugs. At the end of the follow-up

\begin{tabular}{|c|c|c|}
\hline \multicolumn{3}{|l|}{ Table 1|Unit costs } \\
\hline Item & Cost $(£)$ & Source \\
\hline Primary care consultation & 24 & Curtis and Netten ${ }^{22}$ \\
\hline Upper gastrointestinal endoscopy & 275 & NHS 2005 Reference Costs ${ }^{23}$ \\
\hline Outpatient visit medical gastroenterology & 152 & NHS 2005 Reference Costs ${ }^{23}$ \\
\hline Inpatient day & 159 & NHS 2005 Reference Costs ${ }^{23}$ \\
\hline Abdominal ultrasound scan & 67 & NHS 2005 Reference Costs ${ }^{23}$ \\
\hline Cholecystectomy & 1883 & NHS 2005 Reference Costs ${ }^{23}$ \\
\hline Magnetic resonance imaging scan & 313 & NHS 2005 Reference Costs ${ }^{23}$ \\
\hline Oesophageal manometry & 275 & As for endoscopy \\
\hline Surgical procedure/other & 1883 & As for cholecystectomy \\
\hline \multicolumn{3}{|l|}{ Helicobacter pylori tests } \\
\hline Breath test & 22 & Institute of Human Nutrition, University of Southampton \\
\hline Helico G Elisa test & 7.15 & Public Health Laboratory Service \\
\hline Helisal near patient test & 17.77 & Public Health Laboratory Service \\
\hline Histology/culture/rapid urease test & 14.65 & NHS 2005 Reference Costs ${ }^{23}$ \\
\hline
\end{tabular}


Table 2 | Cost of drugs per defined daily dose, standard daily dose, or dispensed unit/pack, as appropriate

\begin{tabular}{lc} 
Item & Cost (£) \\
Amoxicillin & 0.134 \\
\hline Cimetidine & 0.12 \\
\hline Clarithromycin & 1.496 \\
\hline Domperidone & 0.126 \\
\hline Esomeprazole & 0.91 \\
\hline Lansoprazole & 0.24 \\
\hline Mebeverine & 0.242 \\
\hline Metoclopramide & 0.179 \\
\hline Nizatidine & 0.271 \\
\hline Metronidazole & 0.77 \\
\hline Omeprazole & 0.319 \\
\hline Pantoprazole & 0.775 \\
\hline Peppermint oil & 0.304 \\
\hline Rabeprazole & 0.756 \\
\hline Ranitidine & 0.111 \\
\hline HeliClear & 35.01 \\
\hline HeliMet & 33.69 \\
\hline Alginates (strong) & 2.70 \\
\hline Alginates (standard) & 2.25 \\
\hline hydroxide & 1.71 \\
\hline Magnesium trisilicate & 1.00 \\
\hline Sources: Drug Tariff $2005^{24}$ and British National Formulary & \\
\hline
\end{tabular}

Sources: Drug Tariff $2005^{24}$ and British National Formulary $2005 .{ }^{25}$

year, we sent a letter with their result to all patients who had had a follow-up breath test, copied to their general practitioner, and all general practitioners received a summary of the study findings.

\section{Sample size}

We used an economic approach to sample size, using a published discrete event simulation model to determine the likely cost difference, ${ }^{9}$ and combining these data with previous data from randomised controlled trials on variance in costs and an implied maximum willingness to pay of $£ 422$ to determine the minimum required effect. ${ }^{2627}$ Table 3 shows the calculations and sample sizes, which varied from 1040 on effects to 2000 on costs.

\section{Analysis}

We did an intention to treat analysis of intervention versus control for all outcomes. As resource use data were skewed, we used Mann-Whitney tests to compare rates of resource use between the two groups. We compared changes in dyspepsia scores, measured by the change in total score on the short form Leeds dyspepsia questionnaire (out of 32 for both severity and frequency domains) between entry and 12 months, as well as mean satisfaction scores, by using $t$ tests after tests of normality and equal variance. We identified patients with predominant heartburn and predominant epigastric pain and re-examined the primary outcome in each subgroup. We did stochastic cost effectiveness analysis, calculating mean incremental cost effectiveness ratios by using an available case analysis of mean difference in effects and mean difference in costs. ${ }^{28} \mathrm{We}$ used a parametric plot of net monetary benefit versus maximum willingness to pay to display uncertainty around the incremental cost effectiveness ratio. We calculated incremental net benefit (INB) as the net monetary gain, weighting QALYs gained ( $\triangle$ QALY) by the maximum willingness to pay $(\lambda)$ for a QALY, and subtracting the cost difference $(\Delta \mathrm{C}): \mathrm{INB}=\lambda \Delta \mathrm{QALY}$ $-\Delta \mathrm{C}$.

\section{RESULTS}

Participants were randomised between 10 January 2003 and 10 February 2005. We recruited 699 patients from 80 practices; 356 were randomised to empirical treatment and 343 to the test and treat strategy. Nine patients withdrew from the study over the year of follow-up, as they did not wish to be contacted further. We obtained resource use data for $93 \%$ of participants and complete questionnaires for $78 \%$, leaving $76 \%$ of participants available for the economic analysis (fig 1). The groups were well matched with respect to age, sex,

\begin{tabular}{|c|c|c|c|}
\hline Item & Source & Data & $\begin{array}{c}\text { Sample size }(\alpha=0.05, \beta=0.1,75 \% \\
\text { follow-up) }\end{array}$ \\
\hline \multirow{2}{*}{ Costs over one year } & $\begin{array}{l}\text { Mean costs: first order simulation } \\
\text { model }\end{array}$ & $\begin{array}{l}£ 320 \text { test and treat } v £ 274 \text { proton } \\
\text { pump inhibitor }\end{array}$ & \\
\hline & $\begin{array}{l}\text { Variance on costs: previous } \\
\text { randomised controlled trial }\end{array}$ & Standard deviation $£ 320$ & 2000 (power 80\%) \\
\hline Maximum willingness to pay & $\begin{array}{l}\text { Implicit value of preventing relapse } \\
\text { of oesophagitis }\end{array}$ & $£ 422 /$ relapse & \\
\hline Proportion with dyspepsia & $\begin{array}{l}\text { Using } \lambda=\Delta C / \Delta E \text { to calculate } \Delta E \text { from } \lambda \\
\text { and } \Delta C \text {, using proton pump inhibitor } \\
\text { relapse rate of } 70 \%\end{array}$ & $422 / 46=0.11 ; 70 \% \vee 59 \%$ & 1040 \\
\hline Subgroup analysis & $\begin{array}{l}50 / 50 \text { split on predominant } \\
\text { symptom }\end{array}$ & $11 \%$ difference in each group & 2000 \\
\hline EQ-5D QALY & $\begin{array}{l}\text { Population mean QALY } 0.82 \text { (SD } \\
0.17) ; \text { pilot data of patients with } \\
\text { dyspepsia QALY } 0.73 \text { (SD 0.18) }\end{array}$ & $\begin{array}{l}\text { Difference of } 0.05 \text { (representing } \\
\text { ICER of } £ 1000 / Q A L Y \text { ) }\end{array}$ & 800 \\
\hline Satisfaction & 1 point difference & & 1000 \\
\hline
\end{tabular}

$\lambda=$ willingness to pay for a $\mathrm{QALY} ; \Delta \mathrm{C}=$ cost difference; $\Delta \mathrm{E}=$ effect difference; ICER=incremental cost effectiveness ratio; $\mathrm{QALY}=$ quality adjusted life year. 
Table $4 \mid$ Demographics, quality of life, and dyspepsia symptom scores at baseline. Values are numbers (percentages) unless stated otherwise

\begin{tabular}{lcc} 
Characteristics & Test and treat $(\mathrm{n}=\mathbf{3 4 3})$ & Proton pump inhibitor $(\mathrm{n}=\mathbf{3 5 6})$ \\
Male & $166(48)$ & $178(50)$ \\
\hline Smokers (\%) & $99(29)$ & $103(29)$ \\
\hline Dyspeptic symptoms: & $(\mathrm{n}=323)$ & $(\mathrm{n}=335)$ \\
\hline Symptomatic & $321(99.4)$ & $334(99.7)$ \\
\hline Asymptomatic & $2(0.6)$ & $1(0.3)$ \\
\hline Predominant symptom: & & $165(46)$ \\
\hline Epigastric pain & $167(47)$ & $146(41)$ \\
\hline Heartburn & $131(38)$ & $17.0(7.4)(\mathrm{n}=335)$ \\
\hline Mean (SD) SF-LDQ total score (0-32) & $15.5(7.5)(\mathrm{n}=323)$ & $0.744(0.239)(\mathrm{n}=345)$ \\
\hline Mean (SD) EQ-5D utility & $0.743(0.229)(\mathrm{n}=334)$ &
\end{tabular}

SF-LDQ=short form Leeds dyspepsia questionnaire.

smoking status, and EQ-5D utility. No participants were taking non-steroidal anti-inflammatory drugs. However, the baseline mean short form Leeds dyspepsia questionnaire score was greater in the acid suppression group than in the test and treat group $(17.0$ $v$ 15.5) (table 4). One hundred (29\%) patients randomised to test and treat tested positive for $\mathrm{H}$ pylori; 99 of these received eradication treatment and 73 attended for a further breath test. The rate of successful eradication of $\mathrm{H}$ pylori was $78 \%$ (57/73). Of the patients randomised to proton pump inhibitor, none received eradication treatment for proved peptic ulcer disease and $10(2.8 \%)$ were given eradication treatment after subsequent testing for $H$ pylori during the 12 months after study entry, in breach of the protocol.

The test and treat strategy did not significantly reduce the number of patients with symptoms of dyspepsia at one year $-213 / 260(82 \%)$ with symptoms in the test and treat group versus $228 / 275(83 \%)$ in the acid suppression group (absolute risk reduction 1\%, $95 \%$ confidence interval $-5 \%$ to $7 \%)$. Nor did we find a significant difference in quality of life or costs or in either of the pre-specified subgroups (table 5). The incremental cost effectiveness for test and treat versus acid suppression was $£ 1000 /$ QALY. However, given the small differences in both cost and effects, this

Table 5 | Differences in qualityadjusted lifeyears (QALYs), costs, and symptom scores at 12 months

\begin{tabular}{|c|c|c|c|}
\hline & Test and treat & $\begin{array}{l}\text { Proton pump } \\
\text { inhibitor }\end{array}$ & Difference $(95 \% \mathrm{Cl})$ \\
\hline $\begin{array}{l}\text { Difference in SF-LDQ from baseline (all } \\
\text { participants) }\end{array}$ & 7.9 & 8.4 & $0.5(-0.8$ to 1.8$)$ \\
\hline No with symptoms at 12 months & $217 / 265^{\star}(82 \%)$ & $229 / 276^{*}(83 \%)$ & $1.1 \%(-5.4$ to 7.6$)$ \\
\hline $\begin{array}{l}\text { Difference in SF-LDQ from baseline } \\
\text { (epigastric pain predominant patients) } \\
\text { ( } n=245)\end{array}$ & 8.0 & 7.1 & $0.9(-1.2$ to 2.9$)$ \\
\hline $\begin{array}{l}\text { Difference in SF-LDQ from baseline } \\
\text { (heartburn predominant patients) } \\
(n=203)\end{array}$ & 9.5 & 8.5 & $1.0(-1.3$ to 3.2$)$ \\
\hline EQ-5D utility/QALY & 0.834 & 0.830 & $0.004(-0.036$ to 0.044$)$ \\
\hline Mean costs $(£)$ & 132 & 128 & $4(-44$ to 53$)$ \\
\hline
\end{tabular}

SF-LDQ=short form Leeds dyspepsia questionnaire.

*Greater than number on flow chart, as some participants with missing data for scores could be dichotomised into symptoms/no symptoms. estimate is associated with very wide uncertainty. As uncertainty in net health benefit varies with the maximum willingness to pay for a QALY, figure 2 shows a plot of incremental net benefit (mean and 95\% confidence intervals) against maximum willingness to pay. This shows that at no point does test and treat become significantly cost effective compared with initial acid suppression, as the confidence intervals diverge widely as willingness to pay increases. However, the costs for the breath test and eradication of $H$ pylori in the test and treat arm were largely recouped by overall reductions in some other costs (table 6). Fewer ultrasound scans, additional $H$ pylori tests, endoscopies, primary care consultations, outpatient attendances, and inpatient days occurred after test and treat, although only the reduction in $\mathrm{H}$ pylori tests was significant (table 6). It seems that a small number of patients went on to consume further health service resources as a result of their dyspepsia, but that this was reduced in the test and treat arm of the trial. The net effect of this lower consumption of resources was to render test and treat largely resource neutral over the year of follow-up.

The short form Leeds dyspepsia questionnaire scores did not differ significantly between the two groups, and we found no difference within the subgroups of participants with predominant epigastric pain and predominant heartburn at study entry (table 5). Within the test and treat arm itself, we found no significant difference between $H$ pylori positive patients who received eradication treatment and those who tested negative for H pylori (8.4 $v 8.8$ mean change in score). The score for satisfaction with management was similar between the two groups (35/ $45 v 36 / 45$ for primary care management and 37/45 $v$ $36 / 45$ for hospital management). Of the patients who received eradication treatment, 15 reported a metallic taste, 13 had diarrhoea, and 10 had abdominal discomfort. Seven consultations for side effects occurred, but only one patient had to stop the eradication treatment early. No patients died, and no cases of malignancy were detected in the year of follow-up.

The number of participants recruited remained short of our original target of 2000, a target primarily driven by the expected variance in costs. The study has $76 \%$ power on symptoms and $48 \%$ on costs on the basis of an $11 \%$ difference in effect and a $£ 46$ difference in costs. Alternatively, the study has $90 \%$ power to detect a $14 \%$ difference in effect, allowing for 75\% follow-up. As the primary outcome measure in a cost effectiveness study is the incremental cost effectiveness ratio, the change in effect size moves the incremental cost effectiveness ratio that can be confirmed with reliability to $£ 328$ per patient "cured" from the initial $£ 422$ per patient.

\section{DISCUSSION}

The MRC-CUBE study shows that the 2004 NICE guidelines were correct in not recommending an $H$ pylori test and treat strategy over a proton pump inhibitor for the initial management of dyspepsia in 
primary care. This is not because test and treat is less effective or more costly than empirical acid suppression treatment but because it offers no significant advantage at this point in a patient's management. Effects, costs, and satisfaction were similar between the two groups at 12 month follow-up. Although the study did not recruit the number of participants originally planned, post hoc power calculations indicate that the study has adequate power to exclude a clinically important effect. The actual differences in symptoms, QALYs, and costs are $10 \%$ of those sought in the sample size calculations, and an interim analysis, prohibited by the lengthy follow-up, would probably have stopped the study at this point in any case. As we relied on opportunistic recruitment by practices, we could not enhance recruitment by screening for prevalent cases. Although we made very considerable efforts to recruit more centres during the study, this was thwarted by regulatory changes detailed elsewhere. ${ }^{29}$

\section{Comparison with previous studies}

The appropriate design of "pragmatic" cost effectiveness randomised controlled trials has been much debated, as placebo controlled blinded studies may overestimate the real life effects of treatments in nontrial conditions. ${ }^{30}$ As this was a pragmatic study, the trial protocol did not recruit a closely defined subgroup

\begin{tabular}{|c|c|c|}
\hline & Proton pump inhibitor & Test and treat \\
\hline \multicolumn{3}{|l|}{ Defined daily dose per patient } \\
\hline $\begin{array}{l}\text { Proton pump inhibitor prescribed as initial } \\
\text { intervention }\end{array}$ & 30.3 & 30.9 \\
\hline Proton pump inhibitor prescribed in addition & 28.7 & 35.6 \\
\hline $\mathrm{H}_{2}$ receptor antagonist & 3.3 & 5.4 \\
\hline Alginates & 1.6 & 2.4 \\
\hline Antacid & 0.4 & 0.2 \\
\hline Prokinetic agent & 0.7 & 0.4 \\
\hline Antispasmodic agent & 0.1 & 1.1 \\
\hline \multicolumn{3}{|l|}{ Resource use per 1000 participants } \\
\hline Eradication treatment (No of courses) & 48.6 & 321.5 \\
\hline Abdominal ultrasound scan & 27.4 & 3.2 \\
\hline Cholecystectomy & 6.1 & 19.3 \\
\hline Surgical procedure & 3.0 & 6.4 \\
\hline Magnetic resonance imaging scan & 3.0 & 0.0 \\
\hline Barium swallow & 3.0 & 0.0 \\
\hline Oesophageal manometry & 3.0 & 0.0 \\
\hline Upper gastrointestinal endoscopy & 139.8 & 128.6 \\
\hline Barium meal & 0.0 & 0.0 \\
\hline Breath test & 21.3 & 1099.7 \\
\hline HelicoG serology* & 66.9 & 12.9 \\
\hline Helisal serology* & 27.4 & 3.2 \\
\hline Histology & 42.6 & 45.0 \\
\hline HPUK serology* & 36.5 & 9.6 \\
\hline Primary care consultation & 1136.8 & 1125.4 \\
\hline Inpatient days & 18.2 & 9.6 \\
\hline Hospital outpatient appointment & 88.1 & 74.0 \\
\hline
\end{tabular}

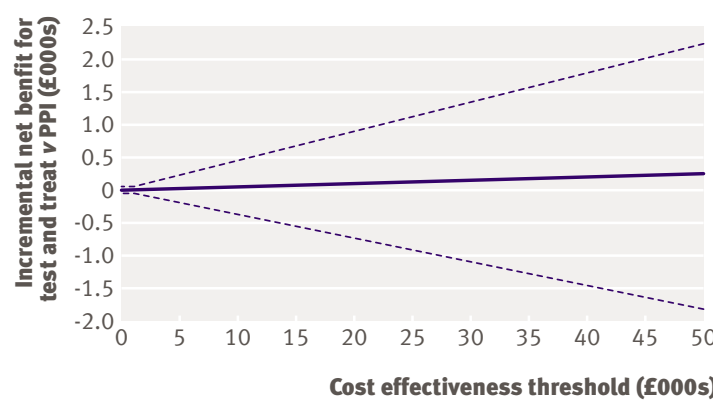

Fig 2 | Incremental net benefit for test and treat versus proton pump inhibitor (PPI) (95\% confidence interval) against maximum willingness to pay

of patients but a broad group with both heartburn and epigastric pain, for whom either test and treat or empirical acid suppression would be used. UK (NICE) guidelines do not recommend the differentiation of uninvestigated upper gastrointestinal problems into dyspepsia and gastro-oesophageal reflux disease on the basis of symptoms. Furthermore, investigation and planned follow-up visits were avoided so as not to distort patterns of routine care. ${ }^{14}$ In 2006 a cluster randomised study in Denmark compared initial proton pump inhibitor, test and treat, and proton pump inhibitor followed by test and treat. ${ }^{31}$ The study, which randomised 222 patients to proton pump inhibitor and 250 to test and treat, found no overall differences in symptoms or costs, but the subgroup of $H$ pylori positive patients given eradication treatment had fewer days of dyspepsia than $\operatorname{did} H$ pylori negative patients. Placebo controlled studies in H pylori positive patients have found a significant benefit in favour of eradication treatment. ${ }^{113}$ This CUBE study and the recent Danish study are consistent with these placebo controlled studies, in that the real life difference in effectiveness between test and treat and initial proton pump inhibitor was less than the difference in efficacy between test and treat and placebo. In terms of guideline development, effectiveness rather than efficacy is important. This study also found no difference between the outcomes for heartburn predominant and epigastric pain predominant patients, supporting the NICE guideline in not separating gastro-oesophageal reflux disease and dyspepsia in uninvestigated patients, although it must be noted that power is lacking on this subgroup analysis.

\section{Study design and validity}

We randomised individual patients to avoid bias resulting from the inability to conceal treatment allocation in a cluster design. One consequence of this is that some contamination of the control group is likely to have occurred. This was limited to $2.8 \%$ and is unlikely to have affected the study result. We chose the eradication regimen as that providing the highest eradication rates for $H$ pylori and omeprazole for the control as the "gold standard" acid suppression treatment. Although not all patients would receive 
proton pump inhibitor initially in normal practice, the use of a proton pump inhibitor in the trial ensures maximum internal validity in that test and treat is compared with standardised "best" acid suppression. Data from the Birmingham endoscopy study indicate that general practitioners are equally likely to prescribe proton pump inhibitors, $\mathrm{H}_{2}$ receptor antagonists, and antacids. ${ }^{26}$ On the question of representativeness, the study was slow to recruit, as described above. We did several audits in practices that had been using the EMIS system with the "pop-up" reminder system operating (half of the recruiting practices). These showed that the slow rate of recruitment was not due to eligible patients being missed but to large numbers of patients with recurrent dyspepsia having already been tested for $H$ pylori. This is important, in that the study is unlikely to be biased by only a selected subgroup being entered.

\section{Value of $H$ pylori eradication}

Eradication of $H$ pylori will largely prevent peptic ulcer disease, ${ }^{32}$ and it may also reduce the risk of development of gastric cancer. ${ }^{33}$ Although the evidence for prevention of gastric cancer is not conclusive, some people would consider it sufficient to warrant early testing and treatment for Hpylori in young patients with dyspepsia in regions where the incidence of gastric cancer is high, such as in China. ${ }^{7}$ Conversely, in areas of very low $H$ pylori prevalence, test and treat could be argued to be largely superfluous. ${ }^{7}$ On this note, we must point out that our trial results pertain to the UK, where the overall prevalence of $H$ pylori is just under $30 \%$. These data may not apply to regions where the prevalence of $H$ pylori is markedly higher or lower. $H$ pylori is virtually disappearing among young people in affluent areas, but in deprived parts of the world and among immigrant communities in the developed world $H$ pylori infection is still as high as $90 \%$. The prevalence of $H$ pylori also has an impact on the choice of non-invasive test. If the prevalence is as low as 10\%, as in affluent areas of the UK, as many as six out of 10 positive serology tests for H pylori will be false positive. This increases the costs of a test and treat strategy, as either unnecessary antibiotic use occurs or a more

\section{WHAT IS ALREADY KNOWN ON THIS TOPIC}

Guidelines recommend that patients with dyspepsia without "alarm" features for upper gastrointestinal cancer are managed with either acid suppression or Helicobacter pylori testing and eradication

For patients with persistent symptoms, testing for and treating $H$ pylori is more cost effective than further management based on an endoscopy

\section{WHAT THIS STUDY ADDS}

Testing for and treating $\mathrm{H}$ pylori at the initial consultation is no more effective at one year than acid suppression alone

The costs of testing for and treating $\mathrm{H}$ pylori at the initial consultation are similar at one year to acid suppression alone, as the initial cost is recovered by cost savings during the year

The decision whether to test for $\mathrm{H}$ pylori initially or only with persistent symptoms remains one to be shared with the individual patient accurate and costly test has to be used. The CUBE study has shown that breath testing with a simple kit (equivalents of which are commercially available on prescription) is quite feasible in primary care. Laboratories may choose to provide stool antigen testing services as an alternative. As a last resort, positive serology tests should be confirmed with a breath or stool test, but as the negative predictive value is reasonable, a negative serology test can be relied on.

\section{Clinical implications}

At the point of failure of initial acid suppression, test and treat is more cost effective than endoscopy based management; a Cochrane meta-analysis of individual patient data showed a clinically insignificant difference in effect and lower costs for test and treat than for prompt endoscopy. ${ }^{8}$ The clinical implications of CUBE need to be considered in the light of this Cochrane review. CUBE found that the costs of initial test and treat were "paid back" by other savings over the first year, so no point exists at which it is "too early" for test and treat to be used and not be at least as cost effective as proton pump inhibitor alone. Waiting until the patient has persistent symptoms clearly favours test and treat over other strategies. At which point between "initial presentation" and "persistent symptoms" test and treat should be used is a matter for discussion with the individual patient. Involvement of patients was the course recommended by the 2004 NICE guidelines, which are supported by this study.

We thank all the staff in the 80 practices that recruited participants, MidReC, the MRC GPRF, and Roger Holder for statistical advice. We also thank the Institute of Human Nutrition, Southampton for the breath test analysis and the pharmacy departments of City Hospital, Birmingham, and Leeds General Infirmary for providing the eradication treatment. Beth Hinks and Vivienne Tsimbili acted as study administrators. Darren Douglas and Hardeep Sandhar supported the MidReC-en system.

Contributors: BCD, PM, RFAL, FDRH, CMCN, SW, and CE contributed to the development of the protocol. BCD, MQ, and ACF did the analyses and interpreted the data. All the authors were involved in the conduct of the study and the revision of the paper. Val Redman managed the study in Birmingham, CE managed it in Nottingham, and AF managed it in Leeds. $M Q$ was the overall study manager. BCD was the principal investigator, drafted the paper, and is the guarantor.

Funding: Medical Research Council, UK (grant No MRC G0001078). The MRC monitored the progress of the trial through a trial steering group chaired by Greg Rubin. The study sponsor was the University of Birmingham.

Competing interests: BCD, RFAL and PM have received speakers' fees from companies that market proton pump inhibitors.

Ethical approval: West Midlands multi-research ethics committee: MREC/ 01/7/49.

Provenance and peer review: Not commissioned; externally peer reviewed.

1 McCormick A, Fleming D, Charlton J. Morbidity statistics from general practice: fourth national morbidity study 1991-1992. London: Office of Population Censuses and Surveys, 1995.

2 Jones RH, Lydeard SE, Hobbs FD, Kenkre JE, Williams El, Jones SJ, et al. Dyspepsia in England and Scotland. Gut 1990;31:401-5.

3 Delaney BC, Moayyedi P. Dyspepsia. In: Stevens A, Raftery J, Mant J, eds. Health care needs assessment 3rd series. Oxford: Routledge, 2006.

4 Delaney BC, Ford AC, Forman D, Moayyedi P, Qume M. Initial management strategies for dyspepsia. Cochrane Database Syst Rev 2005;(4):CD001961.

5 Mason JM, Delaney BC, Moayyedi P, Thomas M, Walt R, North of England Dyspepsia Guideline Development Group. Managing 
dyspepsia without alarm signs in primary care: new national guidance for England and Wales. Aliment Pharmacol Ther 2005;21:1135-43.

6 Malfertheiner P, Megraud F, O'Morain C, Bazzoli F, El-Omar E, Graham D, et al. Current concepts in the management of Helicobacter pylori infection: the Maastricht III consensus report. Gut 2007;56:772-81.

7 Talley NJ, Vakil NB, Moayyedi P. American Gastroenterological Association technical review on the evaluation of dyspepsia. Gastroenterology 2005;129:1756-80.

8 Ford AC, Qume M, Moayyedi P, Arents NL, Lassen AT, Logan RF, et al. Helicobacter pylori "test and treat" or endoscopy for managing dyspepsia: an individual patient data meta-analysis. Gastroenterology 2005;128:1838-44.

9 Delaney BC, Moayyedi P, Deeks J, Innes M, Soo S, Barton PM, et al. The management of dyspepsia: a systematic review. Health Technol Assess 2000;4:1-187.

10 National Institute for Clinical Excellence. The management of dyspepsia in adult patients in primary care. London: NICE, 2004.

11 Chiba N, Veldhuyzen van Zanten SJO, Sinclair P, Ferguson RA, Escobedo S, Grace E. Treating Helicobacter pylori infection in priman care patients with uninvestigated dyspepsia: the Canadian adult dyspepsia empiric treatment-Helicobacter pylori positive (CADETHP) randomised controlled trial. BMJ 2002;324:1012-7.

12 Chiba N, Veldhuyzen Van Zanten SJ, Escobedo S, Grace E, Lee J, Sinclair P, et al. Economic evaluation of Helicobacter pylori eradication in the CADET-Hp randomized controlled trial of $\mathrm{H}$. pyloripositive primary care patients with uninvestigated dyspepsia. Aliment Pharmacol Ther 2004;19:349-58.

13 Stevens R, Baxter G. Benefit of Helicobacter pylori eradication in the treatment of ulcer-like dyspepsia in primary care. Gastroenterology 2001;120(5, suppl 1):A50(260).

14 Manes G, Menchise A, de Nucci C, Balzano A. Empirical prescribing for dyspepsia: randomised controlled trial of test and treat versus omeprazole treatment. BMJ 2003;326:1118-21.

15 Talley NJ, Stanghellini V, Heading RC, Koch KL, Malagelada JR, Tytgat GN. Functional gastroduodenal disorders. Gut 1999;45(suppl 2):I137-42.

16 Drossman DA. The functional gastrointestinal disorders and the Rome III process. Gastroenterology 2006;130:1377-90

17 Talley NJ, Weaver AL, Tesmer DL, Zinsmeister AR. Lack of discriminant value of dyspepsia subgroups in patients referred for upper endoscopy. Gastroenterology 1993;105:1378-86.

18 Adang RP, Ambergen AW, Talmon JL, Hasman A, Vismans JF, Stockbrugger RW. The discriminative value of patient characteristics and dyspeptic symptoms for upper gastrointestinal endoscopic findings: a study on the clinical presentation of 1,147 patients. Digestion 1996;57:118-34.
19 Fraser A, Qume M, Ford A, Moayyedi P, Delaney BC. The short-form Leeds dyspepsia questionnaire (SF-LDQ) validation study. Aliment Pharmacol Ther 2007;25:477-86.

20 Kind P, Dolan P, Gudex C, Williams A. Variations in population health status: results from a United Kingdom national questionnaire survey. BMJ 1998;316:736-41.

21 Baker R. Characteristics of practices, general practitioners and patients related to levels of patients' satisfaction with consultations. BrJ Gen Pract 1996;46:601-5.

22 Curtis L, Netten A. Unit costs of health and social care. Personal Social Services Research Unit: Canterbury,

2005 (available at www.pssru.ac.uk/uc/uc2005 contents.htm)

23 Department of Health. NHS reference costs 2005. London: DH, 2006 (available at www.dh.gov.uk/en/Publicationsandstatistics/ Publications/PublicationsPolicyAndGuidance/DH_4133221).

24 UK Prescription Pricing Authority. Drug tariff 2005. www.ppa.org.uk/ ppa/edt_intro.htm.

25 British Medical Association, Royal Pharmaceutical Society of Great Britain. British national formulary. London: BMA, RPS, 2005 (No 50)

26 Delaney BC, Wilson S, Roalfe A, Roberts L, Wearn A, Redman V, et al. Cost-effectiveness of initial endoscopy for dyspepsia in patients ove the age of 50 years: a randomised controlled trial in primary care. Lancet 2000;356:1965-9.

27 Bate CM. Cost effectiveness of omeprazole in the treatment of reflux oesophagitis. Br J Med Econ 1991;1:53-61.

28 Briggs A, Gray A. Handling uncertainty when performing economic evaluation of healthcare interventions. Health Technol Assess 1999;3(2).

29 Delaney BC. Engaging practitioners in research: time to change the values of practice rather than the way research is carried out? Fam Pract 2007;24:207-8.

30 Roland M, Torgerson DJ. Understanding controlled trials: what are pragmatic trials? BMJ 1998;316:285

31 Jarbol DE, Kragstrup J, Stovring H, Havelund T, Schaffalitzky de Muckadell OB. Proton pump inhibitor or testing for Helicobacter pylor as the first step for patients presenting with dyspepsia? A clusterrandomized trial. Am J Gastroenterol 2006;101:1200-8.

32 Ford A, Delaney B, Forman D, Moayyedi P. Eradication therapy in Helicobacter pylori positive peptic ulcer disease: systematic review and economic analysis. Am J Gastroenterol 2004;99:1833-55.

33 Wong BC, Lam SK, Wong WM, Chen JS, Zheng TT, Feng RE, et al. Helicobacter pylori eradication to prevent gastric cancer in a high-risk region of China: a randomized controlled trial. JAMA 2004;291:187-94.

Accepted: 8 January 2008 